\title{
Seminario de Historia Rural Andina: balance bibliográfico 1966-2016
}

\author{
Rosaura Andazabal Cayllahua \\ randazabalc@unmsm.edu.pe \\ Universidad Nacional Mayor de San Marcos \\ Facultad de Ciencias Sociales \\ Instituto Seminario de Historia Rural Andina
}

\begin{abstract}
SUMILLA:
El presente artículo se aproxima grosso modo a la producción bibliográfica multidisciplinaria que el Seminario de Historia Rural Andina ha realizado en cincuenta años de ininterrumpida labor académica cuyo aporte al campo de las Ciencias Sociales y Humanas es invaluable.
\end{abstract}

Palabras-clave: Seminario de Historia Rural Andina; Historiografía; Ciencias Sociales; Bibliografía.

\section{Seminario de Historia Rural Andina: Bibliographic Balance 1966-2016}

\section{ABSTRACT:}

This article presents an overview of the valuable multidisciplinary scientific contributions that the Seminario de Historia Rural Andina has made for fifty years to the fields of social science and humanities.

Keywords: Seminar of Andean Rural History; Historiography; Social Sciences; Bibliography.

\section{Introducción}

$\mathrm{F}$ undado por el historiador Pablo Macera, el Seminario de Historia Rural Andina (SHRA) de la Universidad Nacional Mayor de San Marcos (UNMSM), inició sus funciones de forma oficial el 10 de marzo de 1966 como un centro de investigación dependiente de la Facultad de Letras y Ciencias Sociales de la UNMSM. ${ }^{1}$ Posteriormente, desde 1972 hasta 1990 estuvo incorporada a la Dirección de Proyección Social. ${ }^{2}$ Después, desde el año 1991 hasta el 5 de junio de 2016, fue un órgano de investigación y asesoría del Rectorado. ${ }^{3}$ Siendo a partir del 6 de junio de 2016 un Instituto de investigación de la Facultad de Ciencias Sociales. ${ }^{4}$ Asimismo, tiene

Acta de fundación del SHRA-UNMSM emitido por la Facultad de Letras y Ciencias Sociales, en la que se resuelve patrocinar dicho centro de investigación.

Resolución Rectoral n. ${ }^{\circ} 36416$ del 06 de junio de 1972

Resolución Rectoral n. ${ }^{\circ} 104636$ del 30 de diciembre de 1991.

Según consta en el actual Estatuto de la UNMSM, promulgado el 6 de junio de 2016, en cumplimiento de la Ley Universitaria n. 30220. La cual reemplaza al Estatuto que regía desde 1984 en la UNMSM. 
bajo su administración el Complejo Arqueológico de Pacopampa, ubicado en el distrito de Querocoto, provincia de Chota, en el departamento de Cajamarca. ${ }^{5}$

En cincuenta años el SHRA ha cumplido una vasta y fecunda labor, cuyo aporte historiográfico a las ciencias sociales y humanas se hace tangible en sus casi 500 publicaciones que, desde una arista multidisciplinaria, transita por todas las etapas de la historia peruana y de parte de Latinoamérica. Trabajo de investigación que el SHRA -con Pablo Macera a la cabeza- se ha cimentado desde su fundación, en el soporte documental acopiado de archivos nacionales y extranjeros. Publicaciones que destacan la figura tantas veces acallada del poblador común que, emerge en el escenario histórico junto a la participación de las autoridades principales. El objetivo es ver, de manera horizontal, el papel que a cada uno de los actores sociales les cupo en la construcción de nuestro patrimonio cultural.

Suma a esa producción bibliográfica, las casi cien exposiciones ${ }^{6}$ de Arte y oralidad del Ande y la Amazonia peruana; de héroes populares cobrando vida bajo la figura del cómic; de coyunturas de gobiernos republicanos graficados a través de la caricatura en la prensa; del arte contemporáneo que ha visibilizado a pintores, escultores, grabadores, fotógrafos y otros personajes asociados a ella; de la vida en las instituciones eclesiásticas; de las tierras de montañas y de la pintura infantil entre varios otros temas. Las exposiciones están dirigidas al público en general, aproximándolos a un amplio espectro de coyunturas históricas, muchas de las cuales se hallan intrínsecamente ligadas a la diversidad de lenguas del Ande y de la Amazonia.

Durante estos cincuenta años de trabajo constante, el SHRA ha estado bajo la dirección del historiador Pablo Andrés Macera Dall'Orso, del arqueólogo Jaime Graciano Miasta Gutiérrez, de la historiadora del Arte Nanda Leonardini Herane, del sociólogo Honorio Sabino Pinto Herrera, de los historiadores Emilio Augusto Rosario Pacahuala y Alex Loayza Pérez. Carlota Alicia Casalino Sen es la primera directora del hoy Instituto Seminario de Historia Rural Andina. Dichas gestiones han tenido tanto lapsos cronológicos como producciones anuales diferenciadas, según desarrollamos en las siguientes páginas de manera individual, y que pueden verse de manera resumida en los gráficos 1 y 2 , los cuales han sido efectuados de acuerdo a un balance estadístico pormenorizado de cada uno de ellos, ${ }^{7}$ a excepción de Loayza que ha estado a cargo del SHRA ocho meses del presente año y Casalino que recién ha dado inicio a su agenda de trabajo.

\section{Balance Bibliográfico 1968-2000: Gestión Pablo Andrés Macera Dall’Orso}

Desde sus inicios, la dinámica del SHRA ha sido acoger las investigaciones de académicos y autodidactas nacionales y extranjeros de las diferentes ramas de las Ciencias Sociales y Humanas, a fin de consolidar un entorno multidisciplinario que permitiera tener una visión global de nuestra sociedad.

La propuesta de investigación planteada por Pablo Macera, definía como eje central a la economía rural para explicar el complejo sistema socioeconómico, político y religioso sobre el cual se construyó históricamente nuestra sociedad. Bajo su dirección se iniciaron varios proyectos de

5 Como consta en el Manual de Organización y Funciones del Seminario de Historia Rural Andina (Oficio n..$^{\circ}$ 01995-R-03 de fecha 26/05/03).

6 De las que se tiene publicados un considerable número de afiches, dípticos, trípticos y catálogos que, dan cuenta del trabajo de investigación, curaduría, museografía, fotografía y montaje efectuado desde 1998 al 2016.

7 La información se obtiene de los catálogos de publicaciones de Andazabal 2006, Pinto 2011, y Candia Quispe 2016. 


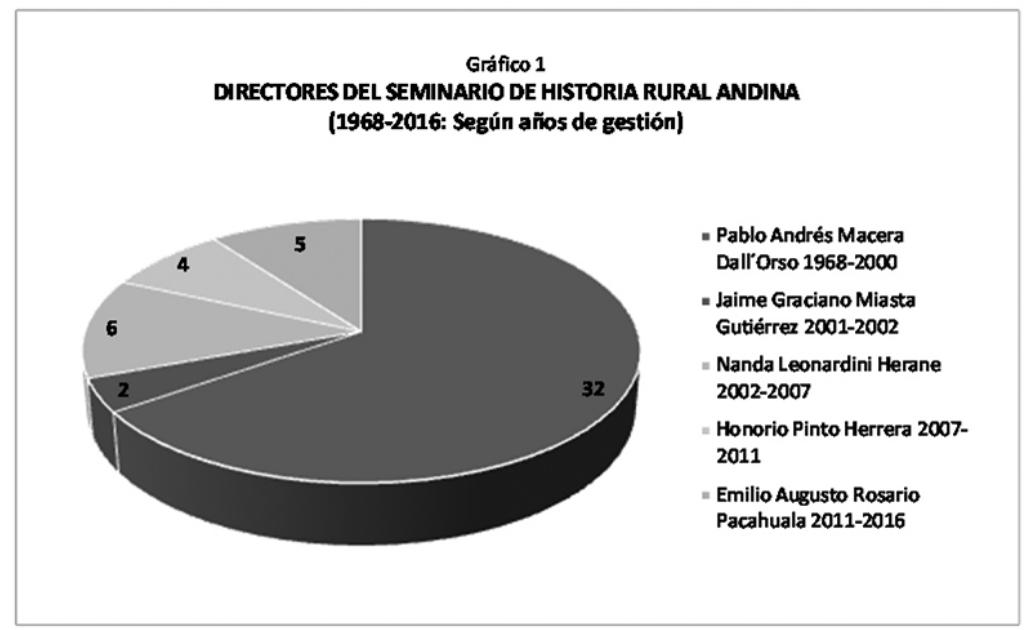

Elaboración: Rosaura Andazabal

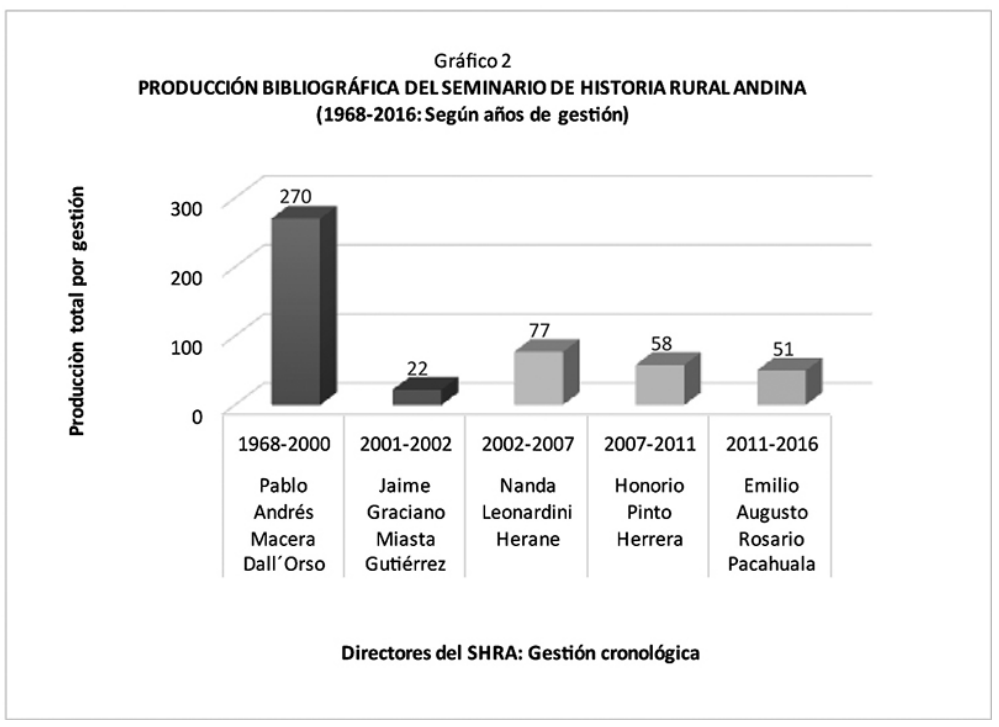

Elaboración: Rosaura Andazabal

investigación conducidos por historiadores y arqueólogos sanmarquinos de la generación de 1960. De allí que el SHRA se organizara en dos frentes principales de investigación, por un lado se desarrollaba el área de Historia y paralela a ella se iniciaron los trabajos de Arqueología en el norte peruano. El área de Historia buscaba renovar la historiografía peruana con investigaciones cimentadas sobre la base de los fondos documentales del Archivo General de la Nación, del Archivo del Ministerio de Trabajo, del Centro de Documentación Agraria, del Ministerio de Fomento, de la Escuela Nacional de Agricultura y de la Compañía Administradora del Guano. ${ }^{8}$ Mientras que las investigaciones del área de Arqueología estuvieron orientadas desde 1966 a 1985 a trabajos de campo cuyo radio geográfico de acción comprendió a disímiles culturas en Cajamarca (Pacopampa, Pandanche, Tacabamba), Alto Amazonas, Junín (Salinas de San Blas, Ushcomachay, Huancayo Alto), Cusco (Vilcabamba: Rosaspata, Nustaishpana y Espíritu Pampa) y Lima (Chancay, Huaro-

$8 \quad$ Macera 1977: LXV-LXVI. 
chirí y Huaura), correspondiente a un período cronológico que abarca desde el Lítico Temprano hasta la etapa Pre-Inca, siendo el Formativo el de mayor producción bibliográfica, sobre todo para Cajamarca. Otras investigaciones de arqueología se han enfocado en el Arte y la Economía. ${ }^{9}$

Durante la primera década del SHRA las publicaciones abarcaron varios ejes de investigación en torno a agricultura, conflictos sociales del sector rural entre los siglos XVI-XX, estadística minera colonial y republicana, reimpresión de libros de historia económica de los siglos XVIII y XIX (Esteves, Copello, Petriconi, Lequanda, Martinet, etc.), e investigaciones de historiadores extranjeros asociadas al mundo rural (David N. Cook, Jhon Fisher y Nicolás Sánchez Albornoz).

Posteriormente, de 1970 en adelante se van perfilando otras propuestas de investigación que incluyen trabajos sociológicos, antropológicos, etnohistóricos y de literatura tradicional andina; siendo el de mayor producción bibliográfica el Área de Antropología, con estudios sobre la producción artesanal de comunidades contemporáneas de la costa y de la sierra peruana. En el área histórica se dio mayor énfasis a los estudios sobre contribuciones indígenas y diezmos; a la par de darse apertura a investigaciones sobre Fuentes de Historia Social Americana relacionadas al Ecuador, Chile, Paraguay, Panamá y Bolivia. En este último país, Pablo Macera seleccionó y recopiló durante varios años fuentes documentales del Archivo Nacional de Sucre. Además de continuarse con los estudios sobre agricultura y tenencia de la tierra, se prosiguió con una serie de publicaciones sobre demografía colonial y republicana hasta la década de $1990 .^{10}$

Paralelamente, desde 1990 se inició un proyecto en torno al fomento y desarrollo de la educación intercultural y bilingüe, a través de la recopilación de la tradición oral del Ande (Ayacucho, Puno, Áncash, Cusco, e hijos de migrantes andinos de escuelas públicas de los conos de Lima Metropolitana) y la Amazonia peruana (Bora, Shipibo Conibo, Asháninka, Nomatsiguenga, entre otros), trabajado por el área de Historia (Rosaura Andazabal y María Belén Soria), con el valioso apoyo externo de la docente Idelsa Mestas Delgado y el antropólogo Javier Macera Urquizo, bajo la dirección de Pablo Macera, que contó con el concurso activo de pintores y narradores autodidactas, quienes han producido relatos orales tradicionales asociados a la concepción propia de las cosmogonías locales de sus lugares de origen. ${ }^{11}$

Toda esa diversidad de investigaciones publicadas bajo la dirección del doctor Pablo Macera, ${ }^{12}$ puede verse en el cuadro 1 que va organizado por temas y disciplinas de las Ciencias Sociales y Humanas. (Ver cuadro 1).

Asimismo, la fluctuación de las publicaciones durante el lapso cronológico de su gestión, si bien queda reflejado en el gráfico 3, ello no necesariamente grafica el trabajo de coyuntura realizado durante los primeros años de funcionamiento del SHRA: las gestiones efectuadas por Pablo Macera, tanto para la adquisición de fondos para Lima, cómo para el mantenimiento, protección, puesta en valor e investigación in situ del Complejo Arqueológico de Pacopampa. Además de haber organizado en simultáneo cátedras de Historia Económica, lecturas de Historia, cursos y conferencias de investigadores extranjeros, a la par de establecer un sistema de comunicaciones con instituciones americanas y europeas. (Ver gráfico 3).

\footnotetext{
$9 \quad$ Andazabal 2006:16.

10 Andazabal 2006: 14

11 Andazabal 2006: 15

12 Quien cesa en su cargo el 27 de julio del 2000, según Resolución Rectoral n. ${ }^{\circ}$ 06994-CR-00 de fecha 03/08/2000. Posteriormente designado como Director Fundador Ad Honorem, según R.R. n. ${ }^{\circ}$ 02219-R-02 de fecha 08/04/2002.
} 


\begin{tabular}{|c|c|c|c|c|c|c|c|c|c|c|c|c|c|}
\hline \multicolumn{14}{|c|}{ 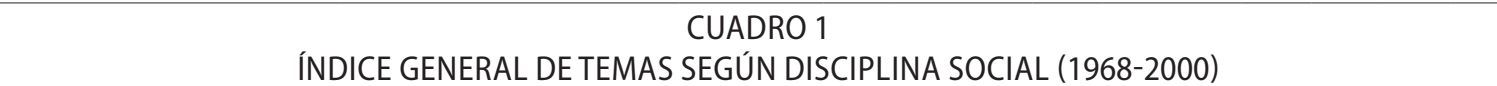 } \\
\hline \multicolumn{3}{|c|}{$\begin{array}{c}\text { TEMAS } \\
\text { (Alfabético/ cronológico) }\end{array}$} & \multicolumn{11}{|c|}{ DISCIPLINAS SOCIALES Y HUMANAS } \\
\hline \multirow[b]{2}{*}{ 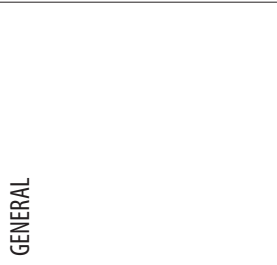 } & \multirow[b]{2}{*}{$\begin{array}{l}\text { 은 } \\
\text { 岕 } \\
\text { 㟧 }\end{array}$} & \multirow[b]{2}{*}{ 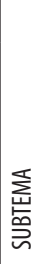 } & \multirow[b]{2}{*}{ 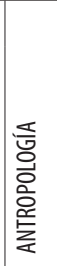 } & \multirow[b]{2}{*}{ 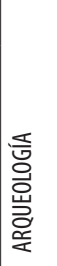 } & \multirow[b]{2}{*}{ 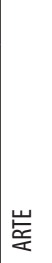 } & \multirow[b]{2}{*}{ 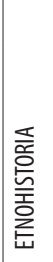 } & \multicolumn{4}{|c|}{ HISTORIA } & \multirow[b]{2}{*}{ 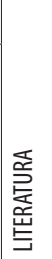 } & \multirow[b]{2}{*}{$\begin{array}{l}\text { 능 } \\
\text { 응 } \\
\text { 응 }\end{array}$} & \multirow[b]{2}{*}{ 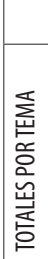 } \\
\hline & & & & & & & § & 高 & 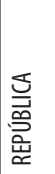 & 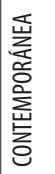 & & & \\
\hline \multirow[t]{8}{*}{ ARTE } & & & & & 2 & & & & & & & 1 & 3 \\
\hline & Folklore & & 3 & & 1 & & & & & & & & 4 \\
\hline & Iconografía/ rituales & & & 5 & & & & & & & & & 5 \\
\hline & Instrumentos musicales & & & 2 & & & & & & & & & 2 \\
\hline & Metalurgia & & & & & & 1 & & & & & & 1 \\
\hline & Pintura mural & & & & & & & 3 & & & & & 3 \\
\hline & Pintura/ Oralidad & & & & & & & & & 6 & & & 6 \\
\hline & Teatro & & & & & & & & 1 & & & & 1 \\
\hline \multirow[t]{2}{*}{ BIBLIOGRAFÍA/HEMEROGRAFÍA } & & & 2 & & & & & 2 & & 2 & & & 6 \\
\hline & Narrativas históricas & & & & & & & & 1 & & & & 1 \\
\hline \multirow[t]{4}{*}{ CONFLICTOS SOCIALES } & & & & & & & & & 4 & & & & 4 \\
\hline & Movimientos campesinos & & & & & & & & 6 & & & & 6 \\
\hline & Movimientos obreros & & & & & & & & 5 & & & & 5 \\
\hline & Pleitos de tierras & & 2 & & & & & 8 & 8 & & & & 18 \\
\hline COSMOGONÍA & & & 2 & & & & & & & & & & 2 \\
\hline CURANDERISMO & & & 1 & & & & & & & & & & 1 \\
\hline \multirow[t]{20}{*}{ ECONOMÍA } & & & 3 & & & 2 & 3 & 5 & 13 & & & & 26 \\
\hline & Agricultura & & 1 & 1 & & & & & 3 & & & & 5 \\
\hline & Algodón & & & & & & & & 3 & & & & 3 \\
\hline & Azúcar & & & & & & & & 5 & & & & 5 \\
\hline & Cascarilla & & & & & & & & 1 & & & & 1 \\
\hline & Caucho & & & & & & & & 1 & & & & 1 \\
\hline & Alfarería & & 3 & 1 & & & & & & & & & 4 \\
\hline & Almojarifazgo & & & & & & & 1 & & & & & 1 \\
\hline & Artesanía & & 4 & & & & & & & & & & 4 \\
\hline & Censos y capellanías & & & & & & & 1 & & & & & 1 \\
\hline & Cofradías & & & & & & & 1 & & & & & 1 \\
\hline & Contribuciones & & & & & & & 6 & 4 & & & & 10 \\
\hline & Diezmos & & & & & & & 5 & & & & & 5 \\
\hline & Haciendas & & & & & & & 2 & & & & & 2 \\
\hline & Minería & & & & & & & 2 & 7 & & & & 9 \\
\hline & Pieles & & & & & & & & 2 & & & & 2 \\
\hline & Precios & & & & & & & 2 & & & & & 2 \\
\hline & Retablos & & 1 & & & & & & & 3 & & & 4 \\
\hline & Textiles & & 4 & & & & & & & & & & 4 \\
\hline & Trabajadores & & & & & & & & 1 & & & & 1 \\
\hline \multirow[t]{3}{*}{ EDUCACIÓN } & Interculturalidad & & 1 & & & & & & & & & & 1 \\
\hline & Juego tradicional & & & & & & & & & 1 & & & 1 \\
\hline & Matemáticas & & & & & & & 2 & & & & & 2 \\
\hline
\end{tabular}




\begin{tabular}{|c|c|c|c|c|c|c|c|c|c|c|c|c|c|}
\hline GEOGRAFÍA & & & & & & & & 7 & 22 & & & & 29 \\
\hline & Población & & 1 & 1 & & & & 3 & 7 & & & 2 & 14 \\
\hline DISTRITOS & & & & & & & & 1 & & & & & 1 \\
\hline INSTITUCIONES & & & & & & & & 1 & & & & & 1 \\
\hline TRADUCCIONES EMILIO CHOY & Traducción del inglés al español & & & 19 & & & & & & & & & 19 \\
\hline \multirow[t]{2}{*}{ LENGUA } & & & & & & & 1 & & & & & & 1 \\
\hline & Oralidad & 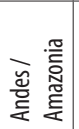 & & & & & & & & & 8 & & 8 \\
\hline MEDICINA & & & 1 & & & & & 4 & 3 & & & & 8 \\
\hline MEMORIAS & & & & & & & & & 2 & & & & 2 \\
\hline \multirow[t]{2}{*}{ PERSONAJES } & & & & & & & & & 1 & & & & 1 \\
\hline & Biografías & & 1 & & & & & & 1 & & & & 2 \\
\hline RELIGIOSIDAD POPULAR & & & 5 & & & & & & & & & 1 & 6 \\
\hline \multirow[t]{4}{*}{ TRABAJO DE CAMPO } & Regiones & శ్ర & & 7 & & & & & & & & & 7 \\
\hline & & $\stackrel{\mathscr{G}}{\text { 乌 }}$ & & 1 & & & & & & & & & 1 \\
\hline & & 言 & & 3 & & & & & & & & & 3 \\
\hline & & 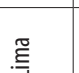 & & 4 & & & & & & & & & 4 \\
\hline TOTALES POR DISCIPLINAS & & & 35 & 44 & 3 & 2 & 5 & 56 & 101 & 12 & 8 & 4 & 270 \\
\hline
\end{tabular}

Elaboración: Rosaura Andazabal

GRÁFICO 3

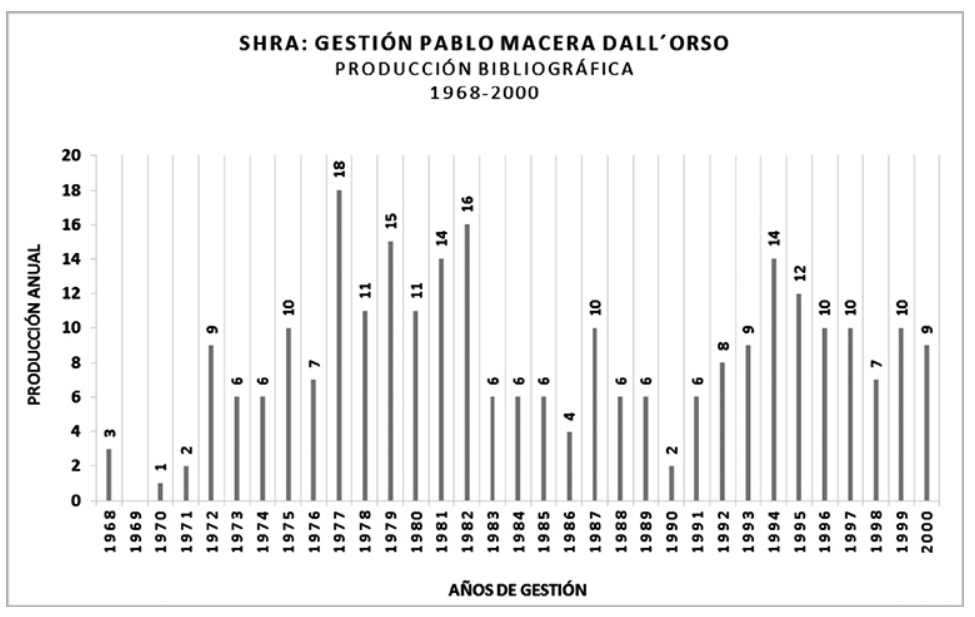

Elaboración: Rosaura Andazabal

Otra explicación de aquellos picos bajos de producción bibliográfica, se halla directamente vinculada a momentos de compilación y trabajo de rastreo documental en archivos históricos peruanos y del extranjero, que por ende prosigue a los picos altos de producción de las décadas de 1970 y 1980 cuando la investigación ha sido procesada. Del mismo modo ocurre para los inicios de 
la década de 1990 en que se inicia un fuerte impulso para el Arte y la Oralidad del Ande y la Amazonia peruana que, a través de proyectos personales como Cuentos Pintados del Perú y Madres y Niñas — bajo la dirección de Pablo Macera — reunió a un conjunto de narradores y pintores autodidactas del Ande y la Amazonia peruana, quienes trabajaron en coordinación con historiadores, antropólogos y lingüistas, para la recopilación, procesamiento y producción de la memoria oral y gráfica de sus respectivos entornos cosmogónicos.

Anexo a este conjunto de acciones académicas, se inicia en esta década la recuperación física de todas las publicaciones que el SHRA hasta entonces había producido, con miras a la formación de la biblioteca institucional que, finalmente se entregó en físico en el año 2006 a la doctora Nanda Leonardini durante su gestión, junto al catálogo de publicaciones que condensa la gestión de Pablo Macera.

Cabe destacar que durante el período de Macera, si bien las publicaciones en su mayoría figuran con las siglas del SHRA y de la UNMSM, existe un buen porcentaje que signa la colaboración y/o apoyo de varias instituciones de nuestra universidad, cómo también figura otro tanto en coedición con instituciones externas, según puede verse en los cuadros $2 \mathrm{a}$ y $2 \mathrm{~b}$.

\section{Balance Bibliográfico 2000-2016}

Ha perdurado hasta el n. ${ }^{\circ} 31$ del año 2009 la serie Lecturas Emilio Choy, iniciada y dirigida desde 1977 por el arqueólogo Jaime Miasta Gutiérrez, la cual perfila una prolija selección de estudios de arqueología, de investigadores de reconocida trayectoria en torno a disímiles temas sobre el Perú, América y otros puntos del orbe, traducidas del inglés al español con el fin de facilitar la lectura de las jóvenes generaciones.

Los estudios sobre Arte y Oralidad del Ande y la Amazonia peruana continuaron el camino trazado en la década precedente por Pablo Macera, a través de su proyecto Cuentos Pintados del Perú habiéndose publicado investigaciones personales en torno a la Amazonia, como las de Víctor Churay (Bora), Roldán Pinedo y Elena Valera (Shipibo); siendo la narrativa y pintura Asháninka de Enrique Casanto Shingari el de mayor producción. En esa línea se ubican también los estudios de antropología e historia desarrollados por Javier Macera Urquizo y María Belén Soria Casaverde, que abordan una temática asociada al curanderismo norteño, cosmovisión, historia republicana, semiótica, simbolismo y nemotecnia. Área que se complementa con los estudios de antropología de Carlos Dávila Herrera sobre Juan Santos Atahualpa y otros conflictos sociales. Mientras que para el Ande, a lo iniciado con el pintor sarhuino Carmelón Berrocal (Ayacucho), el espectro geográfico de estudio se extendió a recoger la oralidad y el Arte Plástico tanto de autodidactas como Félix Condori Vilca (Aimara), Leonidas Zorayda Jara Palacios (Áncash) y Genoveva Núñez Herrera (Cusco); como de escolares de los colegios Johannes Gutenberg (distrito El Agustino) y Enrique López Albújar n. 0502 (Chaclacayo), trabajados por Rosaura Andazabal Cayllahua.

Desde el área de la investigación histórica colonial, republicana y contemporánea entre los años 2000 al 2015 Rosaura Andazabal, Luz Peralta, Miguel Pinto, Alejandro Salinas, María Belén Soria y Santiago Tácunan, han contribuido con nuevos aportes en torno a distintos aspectos socioeconómicos, políticos y religiosos de la historia peruana: criminalidad, instituciones eclesiásticas, composiciones y pleitos de tierras, genealogía, estadística y contribuciones, censos y capellanías, género, ideólogos e iconografía, caudillos, partidos políticos, crisis monetaria, salarios, epistolario Meiggs, guano, prensa, historia distrital, demografía y educación del cono norte de Lima, ferroca- 
CUADRO 2A

SEMINARIO DE HISTORIA RURAL ANDINA - UNMSM

FORMAS DE PRODUCCIÓN: 1968-2015

\begin{tabular}{|c|c|c|c|c|}
\hline Vigencia & Cantidad & SHRA / Series y Colecciones & $\begin{array}{c}\text { SHRA / Coedición Interna, instituciones } \\
\text { UNMSM }\end{array}$ & SHRA / Coedición Externa \\
\hline 1968 & 1 & & Facultad de Letras & \\
\hline $1968-2015$ & 269 & SHRA & & \\
\hline 1971 & 1 & $\begin{array}{l}\text { Centro Peruano de Historia Económica } \\
\text { (CPHE) }\end{array}$ & & $\begin{array}{l}\text { Centro de Estudios de Población y } \\
\text { Desarrollo }\end{array}$ \\
\hline 1971-1972 & 2 & $\begin{array}{l}\text { CPHE } \\
\text { Biblioteca Peruana de Historia } \\
\text { Económica }\end{array}$ & & \\
\hline 1972 & 2 & $\begin{array}{l}\text { Biblioteca Peruana de Historia } \\
\text { Económica }\end{array}$ & & \\
\hline 1972-1979 & 12 & CPHE & & \\
\hline 1973-1975 & 4 & Biblioteca Andina & & \\
\hline 1975 & 1 & $\begin{array}{l}\text { Dirección Universitaria de Biblioteca y } \\
\text { Publicaciones }\end{array}$ & & \\
\hline 1975 & 1 & Serie Huaman Poma n. ${ }^{0} 1$ & Laboratorio de Paleoetnozoología & \\
\hline $1975-1983$ & 6 & Ediciones La Quilca & & \\
\hline 1975-1987 & 25 & & $\begin{array}{l}\text { Dirección Universitaria de Proyección } \\
\text { Social }\end{array}$ & \\
\hline 1977-2009 & 31 & Lecturas Emilio Choy & & \\
\hline 1978-1999 & 20 & $\begin{array}{l}\text { Biblioteca Andina } \\
\text { Fuentes de Historia Social Americana } \\
\text { (FHSA) }\end{array}$ & & \\
\hline $1979-1980$ & 9 & Dirección de Proyección Social & & \\
\hline 1980 & 1 & Centro Andino de Historia & Dirección de Proyección Social & \\
\hline 1980-1984 & 19 & $\begin{array}{l}\text { Centro Andino de Historia } \\
\text { Historia Andina }\end{array}$ & Dirección de Proyección Social & \\
\hline 1981-1982 & 1 & & $\begin{array}{l}\text { Dirección Universitaria de Proyección } \\
\text { Social (DUPS) }\end{array}$ & $\begin{array}{l}\text { Universidad Nacional de Ingeniería } \\
\text { (Centro de Investigaciones Histórico } \\
\text { Tecnológicas) }\end{array}$ \\
\hline 1982 & 1 & CPHE & DUPS & \\
\hline 1983 & 1 & & $\begin{array}{l}\text { Dirección Universitaria de Proyección } \\
\text { Social (DUPS) }\end{array}$ & $\begin{array}{l}\text { Centro de Estudios Rurales Bartolomé } \\
\text { de Las Casas }\end{array}$ \\
\hline 1984 & 1 & Colección Historia Piurana n. ${ }^{0} 1$ & & $\begin{array}{l}\text { Centro de Investigación y Promoción del } \\
\text { Campesinado (CIPCA), Piura }\end{array}$ \\
\hline 1985 & 1 & & & $\begin{array}{l}\text { Seminario de Estudios Antropológicos } \\
\text { de Selva (SEAS) }\end{array}$ \\
\hline 1986 & 1 & & & Convenio Instituto Nacional de Cultura \\
\hline
\end{tabular}

Elaboración: Rosaura Andazabal 
CUADRO 2B

SEMINARIO DE HISTORIA RURAL ANDINA - UNMSM

FORMAS DE PRODUCCIÓN: 1968-2015

\begin{tabular}{|c|c|c|c|c|}
\hline Vigencia & Cantidad & SHRA / Series y Colecciones & $\begin{array}{l}\text { SHRA / Coedición Interna, } \\
\text { instituciones UNMSM }\end{array}$ & SHRA / Coedición Externa \\
\hline 1991-1993 & 4 & & & $\begin{array}{l}\text { Centro de Investigación y Promoción } \\
\text { Amazónica (CIPA) }\end{array}$ \\
\hline 1993 & 1 & & & $\begin{array}{l}\text { CIPA } \\
\text { Archivo General de La Nación (AGN) } \\
\text { Dirección Nacional de Archivo } \\
\text { Histórico (DNAH) }\end{array}$ \\
\hline 1993 y 2015 & 2 & & & $\begin{array}{l}\text { AGN - DNAH, Colección Guillermo } \\
\text { Durand Flórez. }\end{array}$ \\
\hline 1994-1995 & 2 & & & $\begin{array}{l}\text { UNESCO (Encuesta Pintura Mural del } \\
\text { Perú: Coordinación Pablo Macera) }\end{array}$ \\
\hline 1995-1996 & 2 & & Facultad de Ciencias Sociales & \\
\hline 1997 & 3 & & & $\begin{array}{l}\text { Ministerio de Educación (Dirección } \\
\text { Nacional de Formación y Capacitación } \\
\text { Docente) } \\
\text { Deutsche Gesellschaft Für Technische } \\
\text { Zusammenarbeit (GTZ) GMBH }\end{array}$ \\
\hline 1998 & 1 & Serie Economía y Geografía Histórica & & \\
\hline 2001 & 1 & & & $\begin{array}{l}\text { Consejo de Cultura e Identidad Local } \\
\text { de Comas, Calandria (Serie Peruanos } \\
\text { llustres) }\end{array}$ \\
\hline $2001-2002$ & 2 & & $\begin{array}{l}\text { Facultad de Letras y Ciencias } \\
\text { Humanas (Escuela de Arte) }\end{array}$ & \\
\hline 2004 & 1 & & & $\begin{array}{l}\text { Colegio Johannes Gutenberg (El } \\
\text { Agustino) } \\
\text { Tarea Asociación Gráfica Educativa }\end{array}$ \\
\hline 2006 & 1 & & Museo de Arqueología y Antropología & \\
\hline $\begin{array}{l}2004 \text { y } 2010- \\
2013\end{array}$ & 48 & & Centro de Producción Fondo Editorial & \\
\hline $\begin{array}{l}\text { TOTAL publicado } \\
\text { 1968-2015 }\end{array}$ & 478 & & & \\
\hline
\end{tabular}

Elaboración: Rosaura Andazabal

rriles, geografía, curatos y visitas eclesiásticas, mentalidades, fiestas cívicas y religiosidad católica popular, entre varios otros temas.

\section{1-2002: Gestión de Jaime Graciano Miasta Gutiérrez ${ }^{13}$}

Entre los años 2001 y 2002, la dirección del SHRA quedó bajo la batuta del arqueólogo Jaime Miasta, quien si bien le dio mayor énfasis a la publicación de materiales para la investigación arqueológica, fue equitativa la producción en torno a la historia económica colonial y republicana,

13 Director del SHRA-UNMSM, a partir del 29 de julio de 2000 (según Resolución Rectoral n. ${ }^{\circ}$ 043316-R-01, de fecha 09/07/2001), hasta el 30 de mayo de 2002. 
a los conflictos sociales, a manuscritos institucionales y al Arte en general. Fue suyo también el logro de la apertura y creación de la Sala de Exposiciones Colegio Real, que se inauguró con la exposición Madres y Ninas, una colectiva de pintores narradores del Ande y la Amazonia peruana, con la curaduría de Rosaura Andazabal, Javier Macera y Belén Soria y bajo la dirección de Pablo Macera. Esta exposición contó con el auspicio del Rectorado de la UNMSM y fondos de la UNESCO.

Aunque breve, este bienio no cejó en desarrollar el objetivo del SHRA, cuya producción bibliográfica total va anexa en el gráfico 4.

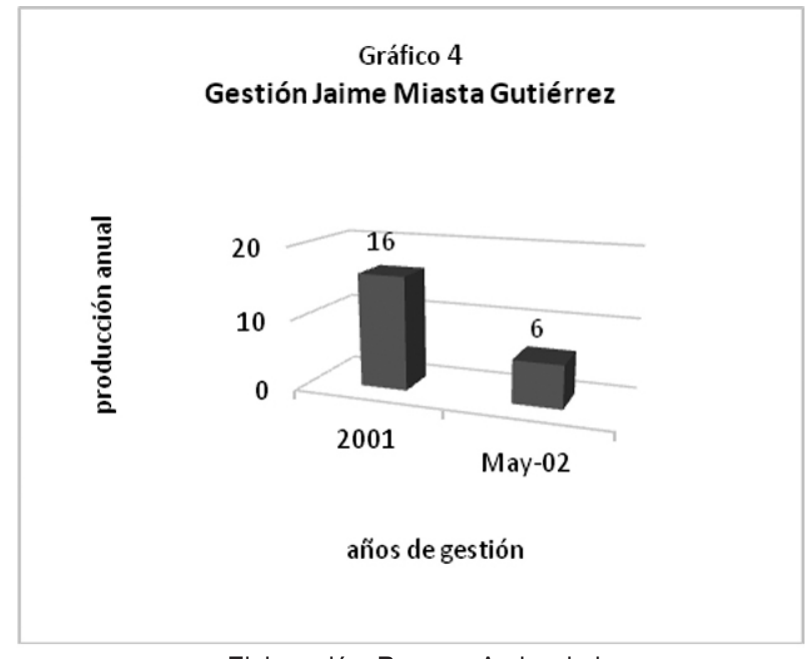

Elaboración: Rosaura Andazabal

\section{2-2007: Gestión de Nanda Leonardini Herane ${ }^{14}$}

Desde junio de 2002 en adelante se posiciona desde la Historia del Arte, diferentes estudios de la etapa republicana y contemporánea con nuevos aportes sobre México y el Perú. Investigaciones que han sido impulsadas por la doctora Nanda Leonardini desde que asumiera la dirección del SHRA, y que hoy se continúa como parte del trabajo multidisciplinario de nuestra institución. En ella han trabajado historiadores del Arte como investigadores del SHRA: Sofía Pachas, Angélica Bráñez, Augusto Gil y actualmente Omar Esquivel; junto a aquellos otros que de manera externa han publicado sus investigaciones como Patricia Mondoñedo y Mary Takahashi entre otros.

La temática de las investigaciones de este período trata sobre pintura mural peruana, escultores, pintores (Gil de Castro, Gamaniel Palomino, Félix Rebolledo, Benjamín Mendoza, Miguel Espinoza Salas, Luis Ugarte, Manuel de Ocaranza y Pablo Roxas), pintura femenina y pintura en miniatura, litografía (Evaristo San Cristóbal), fotografía (Oscar Pacheco) y la crítica de Arte. Asimismo, se continúa con temas históricos al tratar el itinerario de viajes y demografía de la Amazonia peruana, documentación testamentaria y sobre el vestido femenino.

Cabe destacar que si bien dicha dirección mantuvo en cuanto a la temática el perfil multi-

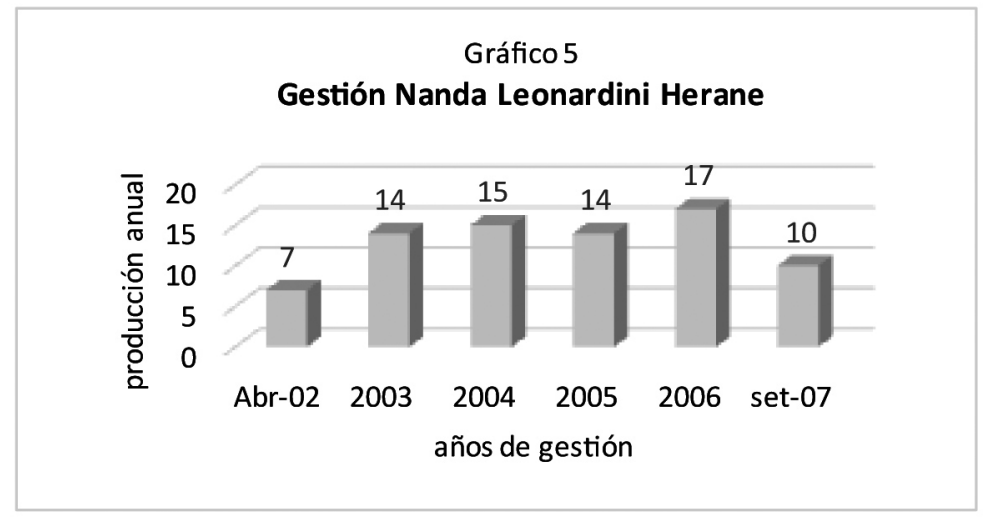

Elaboración: Rosaura Andazabal

14 Es designada mediante Resolución

Rectoral n. ${ }^{\circ}$ 02173-R-02, de fecha 05/04/2002, como Directora del SHRA-UNMSM a partir del 1 de abril de 2002 hasta el 27 de setiembre de 2007. 
disciplinario de las publicaciones precedentes; éstas marcan un quiebre en el aspecto del formato que vira del mimeógrafo en papel bulky, a la impresión digital en papel bond; además del registro formal en la Biblioteca Nacional de las publicaciones con el número de ISBN y el Depósito Legal. Bajo esta dirección se hizo entrega formal de todo el corpus físico de las publicaciones correspondientes a la gestión de Pablo Macera (1968-2000), con lo cual se ha formado la biblioteca de nuestra institución al servicio de investigadores y estudiantes que lo requieran. En este período se inicia también el acopio de obras de Arte, solicitados a cada uno de los artistas que presentan sus individuales y/o colectivas en nuestra Sala de Exposiciones Colegio Real, el cual se mantiene vigente hasta el presente.

La tendencia estadística de la producción bibliográfica anual durante su gestión es como va detallado en el gráfico 5 .

\section{7-2011: Gestión de Honorio Sabino Pinto Herrera ${ }^{15}$}

En octubre de 2007 el SHRA queda bajo la dirección del doctor Honorio Pinto, quien se mantuvo en el cargo hasta mayo del 2011. Él continuó avalando los perfiles precedentes de investigación multidisciplinario, añadiendo su propia cuota inclinada a la investigación republicana y contemporánea de la minería peruana, junto a otros estudios foráneos.

Durante el verano del año 2009 dio su visto bueno para el auspicio y apertura del I Taller de Pintura Infantil dirigido por Juan Zárate, miembro permanente del SHRA.

Asimismo, a partir de febre-

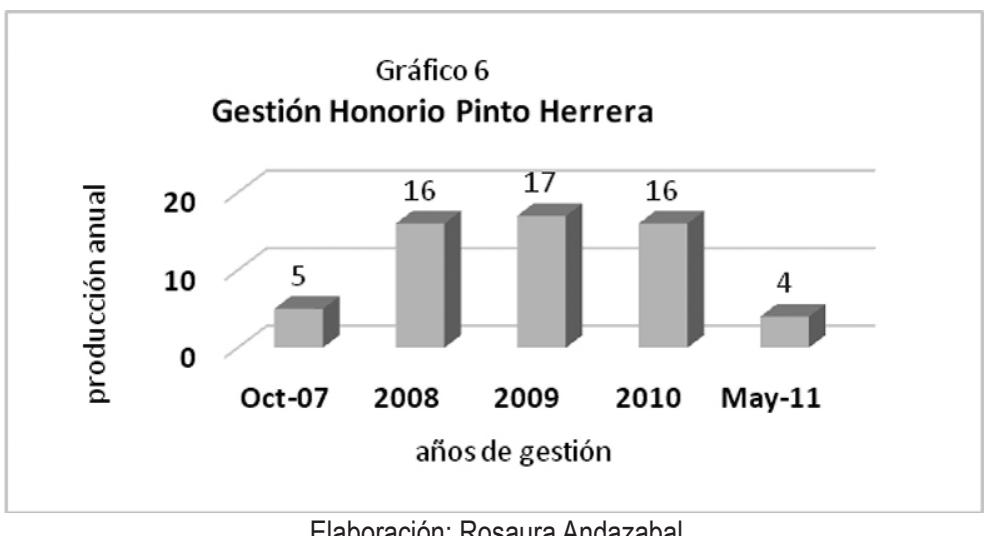

Elaboración: Rosaura Andazabal ro de 2010, con la publicación $L a$ cultura en el discurso de los dirigentes universitarios de la izquierda sanmarquina: 1995-2000, de Omar Yalle Quincho, las publicaciones del SHRA inician una coedición con el Centro de Producción del Fondo Editorial, de la UNMSM. Los estándares de productividad bibliográfica de la gestión de la gestión del doctor Pinto se encuentra en el gráfico 6.

\section{1-2016: Gestión de Emilio Augusto Rosario Pacahuala ${ }^{16}$}

Desde junio de 2011 hasta el 19 de enero de 2016 la dirección del licenciado Emilio Rosario ha continuado con las líneas de investigación del SHRA, incorporando la organización de los Congresos de Historia y Cultura, y la publicación de su respectivas actas, donde es posible ver una malla

15 Designado Director del SHRA-UNMSM a partir del 28 de setiembre de 2007, según Resolución Rectoral n. ${ }^{\circ}$ 05332-R-07, hasta el 14 de junio de 2011.

16 Según Resolución Resolución n. ${ }^{\circ}$ 02995-R-11, de fecha 15/06/2011 es designado Director del SHRA-UNMSM hasta el 19 de enero de 2016. 
temática diversa de investigadores foráneos al SHRA.

$\mathrm{Su}$ dirección mantuvo tanto el registro de las publicaciones en la Biblioteca Nacional, como la coedición con el Centro de Producción del Fondo Editorial de la UNMSM hasta el año 2013, que culmina con el libro de Rolando Pachas Apuntes de Historia del Perú. Siglos XIX-XX (1884-1930). Desde

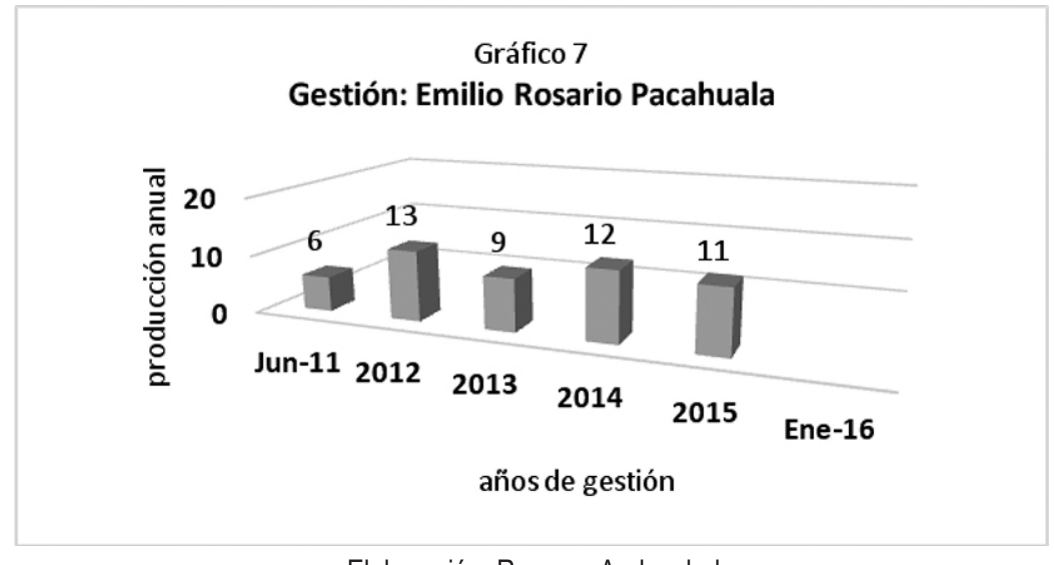

Elaboración: Rosaura Andazabal allí hasta diciembre de 2015 todas las publicaciones figuran sin el número ISBN, con tan sólo el Depósito Legal, a excepción de algunos libros cuyos autores han logrado hacerlo a través de otras instituciones que coeditan la publicación. La estadística anual de producción bibliográfica para dicho lapso puede verse en el gráfico 7.

\section{Enero-agosto 2016: Gestión Alex Loayza Pérez ${ }^{17}$}

Desde enero del presente año asumió el cargo el magister Alex Loayza Pérez que ha coincidido con el cincuenta aniversario de nuestra institución. Coyuntura que ha manejado a través de un plan de trabajo que ha tenido a bien continuar con los cursos-talleres de verano ya preestablecidos como el VIII Taller de Pintura Infantil organizado y dirigido por el artista plástico Juan Zárate Cuadrado y el ler. Curso-Taller: Hatun Misayuq Ruwakusunchik / Hagamos un Cajón San Marcos estructurado y dirigido por Rosaura Andazabal, que ha contado con el valioso aporte en la parte práctica por la retablista cusqueña Genoveva Núñez Herrera y la teoría dictada por Andazabal.

Asimismo, Loayza ha continuado en los meses de su gestión con la programación de varias publicaciones ya previstas para el presente año, las que ha organizado en dos líneas de edición. En primer lugar, la publicación de fuentes documentales e impresos en la colección Fuentes para la Historia Andina -que retoma a una de las series que se manejaba en el SHRA bajo la gestión de Pablo Macera- que se inician con la edición de Antonio Coello de la Memoria de la fiebre amarilla sufrido en Lima en el año de 1868. Escrito por el Dr. Cecilio Velásquez; y los Testamentos inéditos de los caciques del valle del Mantaro (Sierra Central del Perú) Siglos XVII-XVIII, editados por Carlos Hurtado y Víctor Solier. Por otro lado, la segunda línea de edición denominada Cuadernos de investigación se ha iniciado con el trabajo de Carina Sotelo, titulado El Manto Blanco de Paracas: Testimonio de la Cosmovisión del Hombre de Paracas.

Cumpliendo su agenda, Loayza en un coordinado trabajo con todo el plantel del SHRA inaugura el 10 de marzo la muestra titulada Seminario de Historia Rural Andina. 50 años. Actividades ejecutadas como parte de las celebraciones por el cincuentenario del SHRA, que ha continuado el 19 de mayo del presente año, con la participación en pleno del área de investigación en el XXV COLOQUIO HISTORIA DE SAN MARCOS. 50 Aniversario del Seminario de Historia Rural

17 Designado Director del SHRA-UNMSM del 20 de enero (Resolución Resolución n. ${ }^{\circ}$ 00150-R-16, 20/01/2016) hasta el 14 de agosto de 2016. 
Andina y del Centro de Estudiantes de Historia,${ }^{18}$ donde en varias mesas se dio a conocer el aporte historiográfico de nuestro centro de investigación a las Ciencias Sociales y Humanas.

$\mathrm{Al}$ presente se halla en proceso de producción el Catálogo de Aniversario que condensa la temática perfilada en la exposición, y junto a ella el nacimiento de este primer número de la Revista del Instituto del Seminario de Historia Rural Andina.

En paralelo, se han perfilado los proyectos de investigación individual de larga duración, proyectada para los años 2017 - 2019, que si bien es de libre elección temática, todos conforman una unidad de trabajo en torno al Bicentenario de la Independencia.

\section{Agosto 2016: Carlota Alicia Casalino Sen ${ }^{19}$}

La reciente gestión continúa lo ya trazado en la agenda de trabajo precedente e inicia una nueva etapa como Instituto Seminario de Historia Rural Andina (ISHRA). El 20 de setiembre del año en curso se presentó las tres publicaciones antes señaladas para la gestión de Alex Loayza, en el marco inaugural de las celebraciones por el Aniversario de la Facultad de Ciencias Sociales, que continuó hasta el día 23 con la exposición de algunas publicaciones de nuestra institución a toda la comunidad universitaria.

Asimismo, la doctora Carlota Casalino ha iniciado el nexo vinculante entre los miembros del ISHRA y la Facultad de Ciencias Sociales, a través de una primera reunión con los profesores (13 de setiembre) con los alumnos y egresados a través del taller Balance y perspectiva del Seminario de Historia Rural Andina y su proceso de inserción en la Facultad de Ciencias Sociales, dictado el día 4 de octubre y el 7 de noviembre por todo el plantel del ISHRA.

En lo que queda del año se han organizado, reuniones y talleres para profesores, alumnos y toda la comunidad universitaria.

\section{Reflexiones finales}

El hoy Instituto Seminario de Historia Rural Andina fue un proyecto planeado por Pablo Macera mientras seguía estudios de doctorado en Francia, por la década de 1950. Fue creado como un centro de investigación de perfil multidisciplinario que ha priorizado estudios de diversa temática sobre el mundo rural, en un eje transversal que abarca desde la prehistoria hasta la historia contemporánea de todo el territorio peruano que, incluye algunos espacios de Latinoamérica. A las que se han ido sumando con el tiempo investigaciones sobre las zonas urbanas. De otra parte, en el SHRA, Pablo Macera ha brindado oportunidades laborales en sentido horizontal, tanto a egresados sanmarquinos como a aquellos procedentes de otras universidades, donde ha buscado complementar la formación con la que ya venían los investigadores, adiestrándolos en el rastreo, organización y análisis de las fuentes documentales, así como en la ejecución final de la estructura y redacción de la obra. El producto de este trabajo, continuado por las siguientes direcciones, a los

18 Organizado por la Escuela Académico Profesional de Historia; por el Centro de Estudios Historia; por la Dirección de Turismo del Centro Cultural de San Marcos; por la Cátedra Maticorena y por el Seminario de Historia Rural Andina. Realizado en la Casona de San Marcos y en el auditorio "José María Arguedas”, de la Facultad de Ciencias Sociales, de la UNMSM, los días 19 y 20 de mayo de 2016.

19 Encargada de la Dirección del Instituto SHRA, de la Facultad de Ciencias Sociales, mediante Resolución Decanal n. 0593-D-FCCSS-2016, a partir del 12 de agosto del presente año. 
cincuenta años de la existencia del SHRA se ha materializado en las publicaciones que este artículo ha presentado de forma general.

\section{Bibliografía}

Andazabal Cayllahua, Rosaura (2006). Publicaciones. Seminario de Historia Rural Andina (19682000). Lima: Seminario de Historia Rural Andina, Universidad Nacional Mayor de San Marcos.

Candia Quispe, Yolanda (2016). Publicaciones del SHRA: 2011-2015. (Catálogo inédito) Lima: Seminario de Historia Rural Andina, Universidad Nacional Mayor de San Marcos.

Macera Dall’Orso, Pablo (1977). Trabajos de Historia. Tomo I. Lima: Instituto Nacional de Cultura.

Pinto Herrera, Honorio (2011). Seminario de Historia Rural Andina. Publicaciones 2001-2010. Lima: Seminario de Historia Rural Andina, Universidad Nacional Mayor de San Marcos.

Fecha de recepción: 29/VIII/2016

Fecha de aceptación: 30/IX/2016 